\title{
Controlling Drones from 5G Networks
}

\author{
János Czentye, János Dóka, Árpád Nagy, \\ László Toka, Balázs Sonkoly \\ MTA-BME Network Softwarization Research Group \\ \{czentye,sonkoly\}@tmit.bme.hu
}

\begin{abstract}
Envisioned 5G applications are key drivers of the evolution of network and cloud architectures. These novel services pose several challenges on the underlying infrastructure in terms of latency, reliability or capacity, just to mention a few. Controlling or coordinating both indoor and outdoor drones from future networks is a potential application with significant importance. Today's technologies addressing network softwarization, such as Software Defined Networking (SDN) and Network Function Virtualization (NFV), enable a novel way to create and provision such services. In this demonstration, we showcase an Industry 4.0 use-case including a local factory equipped with drones and local cloud and network facilities connecting to remote cloud resources. The envisioned service is realized by a Service Function Chain (SFC) consisting of network functions and logical connections between them with special requirements. In addition, the envisioned service is integrated with our multi-domain resource orchestration system and as a result, it can be controlled, deployed and monitored from that framework. The use-case and the demo well illustrate several aspects and challenges which should be addressed by future $5 \mathrm{G}$ systems.
\end{abstract}

\section{CCS CONCEPTS}

- Networks $\rightarrow$ Programmable networks; Layering;

\section{KEYWORDS}

NFV; SDN; multi-domain orchestration; SFC; 5G; drones

\section{ACM Reference Format:}

János Czentye, János Dóka, Árpád Nagy, László Toka, Balázs Sonkoly and Róbert Szabó. 2018. Controlling Drones from 5G Networks. In

\footnotetext{
Permission to make digital or hard copies of all or part of this work for personal or classroom use is granted without fee provided that copies are not made or distributed for profit or commercial advantage and that copies bear this notice and the full citation on the first page. Copyrights for components of this work owned by others than ACM must be honored. Abstracting with credit is permitted. To copy otherwise, or republish, to post on servers or to redistribute to lists, requires prior specific permission and/or a fee. Request permissions from permissions@acm.org.

SIGCOMM Posters and Demos '18, August 20-25, 2018, Budapest, Hungary

(c) 2018 Association for Computing Machinery.

ACM ISBN 978-1-4503-5915-3/18/08. . \$15.00

https://doi.org/10.1145/3234200.3234244
}

\author{
Róbert Szabó \\ Ericsson Research, Hungary \\ robert.szabo@ericsson.com
}

SIGCOMM Posters and Demos '18: ACM SIGCOMM 2018 Conference Posters and Demos, August 20-25, 2018, Budapest, Hungary. ACM, New York, NY, USA, 3 pages. https://doi.org/10.1145/3234200. 3234244

\section{INTRODUCTION}

Drones are by design made to be piloted from the ground control stations and many such control stations are available with an open source code base. Transforming the control process into software and adding the capability to the network to be in charge of controlling or coordinating drones are challenging tasks with significant future potential. A good example is an Industry 4.0 factory premises equipped with indoor drones (e.g., for moving accessories) controlled by software components running in the cloud (local or remote).

SDN, NFV and SFC are key enablers of future network services and applications, and they are essential building blocks of 5G systems. SDN decouples network control and forwarding functions and addresses the softwarization of the control plane. It makes the network directly programmable and provides a resource abstraction for services. NFV aims at transforming traditional hardware based network services into software based solutions consisting of software components running on general purpose hardware platforms. SFC is a simple but powerful technique for describing complex services built from smaller Virtual Network Functions (VNFs) which can be connected to each other in a flexible and programmable way. These techniques can make $5 \mathrm{G}$ capable of realizing the envisioned drone control applications.

In this demonstration, we present an Industry 4.0 application controlling indoor drones in a factory. The application is implemented as an SFC which is orchestrated by our multidomain resource orchestrator named ESCAPE [2]. ESCAPE is capable of setting up and configuring service chains on demand, mapping VNFs into physical resources (local or remote ones in the current example), steering traffic according to chains' policies and requirements, and providing real-time management information on running VNFs.

\section{OUR USE-CASE AND DEMO SETUP}

The goal of our demonstration is to provide a scenario where delay sensitive processes are controlled by a software based 


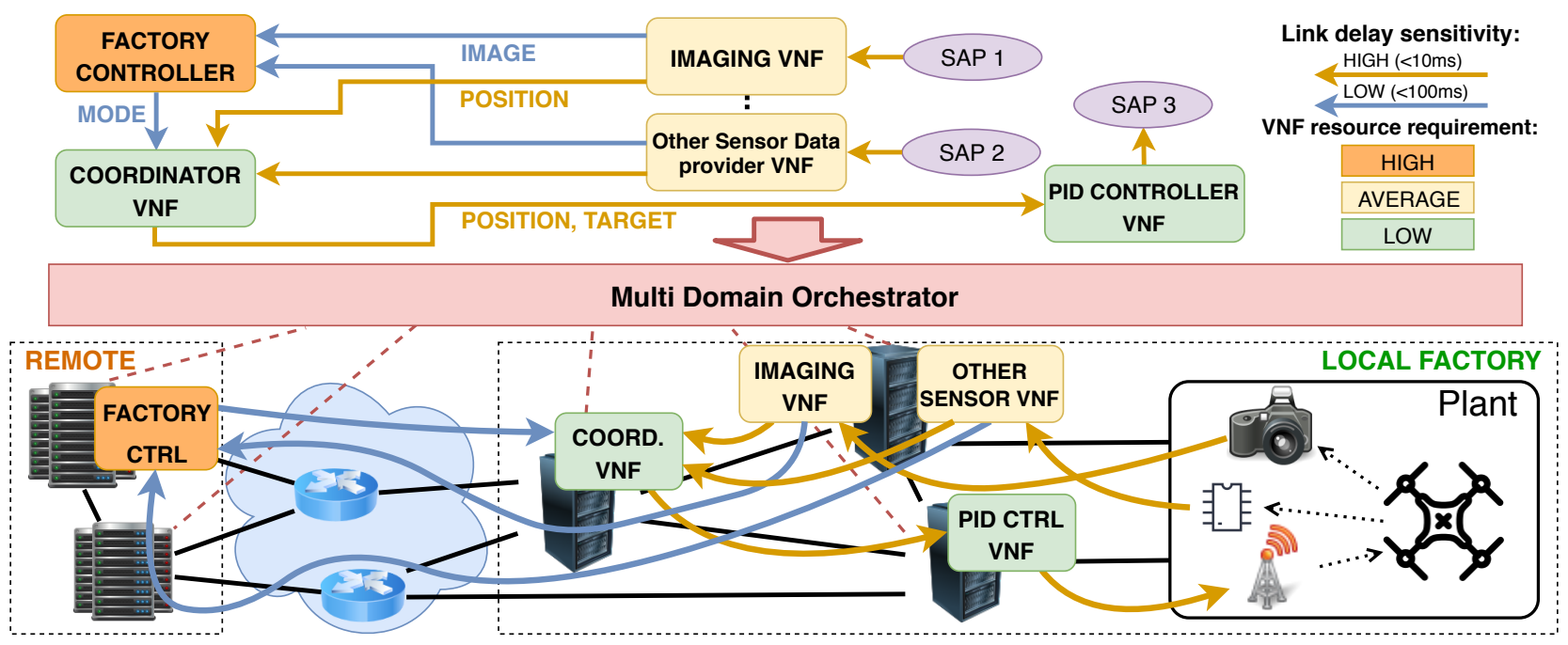

Figure 1: The service chain describing the drone service and the deployment on multi-domain infrastructure.

service running on a distributed cloud environment encompassing local (edge) and remote (cloud) resources. Our highlevel setup and the infrastructure are shown in the bottom part of Fig. 1. On the one hand, the local factory can be considered as the edge where typically limited computing capacity is available with low latency. On the other hand, in remote cloud domains, we can access a huge amount of compute resources with the cost of larger delay. In the factory, the controlled plant encompasses an $i$ ) indoor drone viewed by a $i$ ) fix camera continuously streaming images, iii) a radio device sending control signals to the drone, and $i v$ ) additional sensors. This plant is to be controlled by on-demand deployed software modules or VNFs running as Docker containers.

The upper part of Fig. 1 shows our virtualized service realizing the drone control in the factory. More exactly, it describes the SFCs, the connections between the VNFs and requirements such as, compute flavor required by a VNF, and latency requirements of logical connections indicated by different colors of the graph. The service is attached to the plant at service access points denoted by SAPs.

Imaging $V N F$ is responsible for the imaging function, i.e., it translates the images from the camera in the plant to the position coordinates of the drone. The algorithm which detects the drone is adopted from [1]. Coordinator VNF gathers the data sent by the imaging VNF, and other sensors if they are present, then translates these coordinates to the process variable for the PID controller. This VNF also provides the target coordinates for the PID controller. Factory Controller is the top-level controller of the service and it provides a management and monitoring interface. This function sends the desired flight modes to the coordinator. The video stream from the camera and other sensor data are available here.
The factory controller is not sensitive to the latency and the delay fluctuation because it only controls the operation in a larger time scale. However, if it has to make decisions based on a lot of sensor data then it may require large computing capacity. PID Controller VNF includes four PID controllers controlling each coordinate of the aircraft, respectively. It calculates the appropriate command signal from the error between the target and the actual coordinates of the drone for each coordinate, then converts the command signals to the appropriate remote control signals which control the throttle, rudder, aileron and elevator of the drone.

The control plane of the system is comprised of our multidomain orchestrator, i.e., ESCAPE, and dedicated domain orchestrators running in the resource domains, respectively. Control channel connections are shown by dashed red lines in Fig. 1. The service request is the input of ESCAPE, which calculates an appropriate embedding taking the given requirements into consideration and deploys the result.

During the course of the demo, we send a configurable service request to ESCAPE which will be deployed according to the requirements. Local notebooks provide Docker hosts as edge execution environments while an OpenStack system running in our laboratory plays the role of the remote cloud. After a successful deployment, the flight of the drone can be controlled from the factory controller by high level commands such as, take off, land, fly in a specific pattern, and the impact of the specified requirements and the current deployment can be illustrated. Learn more:

https://sb.tmit.bme.hu/mediawiki/index.php/Drone-5G

Acknowledgments This research was supported by H2020-ICT-2014 project 5GEx (grant agreement no. 671636), which is partially funded by the European Commission. 


\section{REFERENCES}

[1] 2016. Teach your PC to fly a Mini-Drone! (2016). https://www. makehardware.com/2016/04/24/teach-your-pc-to-fly-a-mini-drone/

[2] Attila Csoma, Balázs Sonkoly, Levente Csikor, Felicián Németh, Andràs Gulyas, Wouter Tavernier, and Sahel Sahhaf. 2014. ESCAPE: Extensible service chain prototyping environment using mininet, click, netconf and pox. In ACM SIGCOMM Computer Communication Review, Vol. 44. ACM, 125-126. 